\title{
Assessment of Extrapolation Relations of Displacement Speed for Detailed Chemistry Direct Numerical Simulation Database of Statistically Planar Turbulent Premixed Flames
}

\author{
Nilanjan Chakraborty ${ }^{1} \cdot$ Alexander Herbert $^{2}$ - Umair Ahmed ${ }^{1} \cdot$ Hong G. Im $^{3}$ • \\ Markus Klein² (D)
}

Received: 28 December 2020 / Accepted: 6 July 2021 / Published online: 10 August 2021

(c) The Author(s) 2021

\begin{abstract}
A three-dimensional Direct Numerical Simulation (DNS) database of statistically planar $\mathrm{H}_{2}$ - air turbulent premixed flames with an equivalence ratio of 0.7 spanning a large range of Karlovitz number has been utilised to assess the performances of the extrapolation relations, which approximate the stretch rate and curvature dependences of density-weighted displacement speed $S_{d}^{*}$. It has been found that the correlation between $S_{d}^{*}$ and curvature remains negative and a significantly non-linear interrelation between $S_{d}^{*}$ and stretch rate has been observed for all cases considered here. Thus, an extrapolation relation, which assumes a linear stretch rate dependence of density-weighted displacement speed has been found to be inadequate. However, an alternative extrapolation relation, which assumes a linear curvature dependence of $S_{d}^{*}$ but allows for a non-linear stretch rate dependence of $S_{d}^{*}$, has been found to be more successful in capturing local behaviour of the density-weighted displacement speed. The extrapolation relations, which express $S_{d}^{*}$ as non-linear functions of either curvature or stretch rate, have been found to capture qualitatively the non-linear curvature and stretch rate dependences of $S_{d}^{*}$ more satisfactorily than the linear extrapolation relations. However, the improvement comes at the cost of additional tuning parameter. The Markstein lengths $L_{M}$ for all the extrapolation relations show dependence on the choice of reaction progress variable definition and for some extrapolation relations $L_{M}$ also varies with the value of reaction progress variable. The predictions of an extrapolation relation which involve solving a non-linear equation in terms of stretch rate have been found to be sensitive to the initial guess value, whereas a high order polynomial-based extrapolation relation may lead to overshoots and undershoots. Thus, a recently proposed extrapolation relation based on the analysis of simple chemistry DNS data, which explicitly accounts for the non-linear curvature dependence of the combined reaction and normal diffusion components of $S_{d}^{*}$, has been shown to exhibit promising predictions of $S_{d}^{*}$ for all cases considered here.
\end{abstract}

Keywords Density-weighted displacement speed $\cdot$ Curvature $\cdot$ Stretch rate $\cdot$ Extrapolation relation $\cdot$ Markstein length

Markus Klein

markus.klein@unibw.de

Extended author information available on the last page of the article 


\section{Introduction}

The flame propagation in premixed combustion is well-known to be affected by the flame surface curvature and stretch rate (Klimov 1963; Markstein 1951; Karlovitz et al. 1953; Istratov and Librovich 1969; Matalon and Matkowsky 1982; Wu and Law 1984; Kelley and Law 2009; Chen 2011; Kelley et al. 2011; Wu et al. 2005), and an extensive review of the subject is provided by Lipatnikov and Chomiak (2005). In premixed turbulent combustion, the flame propagation is quantified by the flame displacement speed $S_{d}$, which represents the instantaneous speed at which a given scalar isosurface moves normal to itself with respect to the background fluid motion. The density change gives rise to a change in $S_{d}$ within the flame but in a planar unstrained laminar premixed flame, the densityweighted displacement speed $S_{d}^{*}=\rho S_{d} / \rho_{0}$ (where $\rho$ and $\rho_{0}$ are instantaneous gas density and unburned gas density, respectively) remains identical to the unstrained laminar burning velocity $S_{L}$. However, this equality (i.e. $S_{d}^{*}=S_{L}$ ) is invalid for stretched and curved premixed flames (Giannakopoulos et al. 2015; Chakraborty and Cant 2011; Sabelnikov et al. 2017) and the statistics of $S_{d}^{*}$ are often necessary in the level-set (Peters 2000) and Flame Surface Density (FSD) (Pope 1988) based modelling methodologies. The importance of displacement speed in the closure of the FSD transport equation were discussed in detail by Hawkes and Cant (2001), and Chakraborty and Cant (2009) demonstrated that the effects of local flame curvature $\kappa_{m}$ and flame stretch rate $K=\left(a_{T}+2 S_{d} \kappa_{m}\right)$ (where $a_{T}$ is the tangential strain rate) on the density-weighted displacement speed $S_{d}^{*}$ need to be addressed for accurate closure of the FSD transport equation in the context of Large Eddy Simulations (LES). One of the simplest extrapolation relations expresses $S_{d}^{*}$ as a linear function of $K$ with the help of a length $L_{M}$ known as the Markstein length (Wu and Law 1984). This relation will henceforth be referred to as the linear stretch (LS) extrapolation. Several (for a review see Lipatnikov and Chomiak (2005)) experimental (Kelley and Law 2009; Karpov et al. 1997) or Direct Numerical Simulation (DNS) (Dave et al. 2020; Chen and Im 1998; Chakraborty et al. 2007) studies demonstrated non-linear $K$ dependence of $S_{d}^{*}$, and accordingly Kelley and Law (2009) proposed a quasi-steady non-linear extrapolation relation, which will henceforth be referred to as the NQ model (see Table 1). The NQ model was derived based on the study of Ronney and Sivashinsky (1989) on weakly stretched premixed flames, and this extrapolation relation remains valid not only for near unity Lewis

Table 1 Summary of extrapolation relations

\begin{tabular}{|c|c|c|}
\hline Model & Description & \\
\hline LS & $\begin{array}{l}\text { Linear model based on stretch } \\
\text { (Wu and Law 1984) }\end{array}$ & $S_{d}^{*}=S_{L}-L_{M} K$ \\
\hline NQ & $\begin{array}{l}\text { Quasi-steady non-linear model } \\
\text { (Kelley and Law 2009) }\end{array}$ & $\left(S_{d}^{*} / S_{L}\right)^{2} \ln \left(S_{d}^{*} / S_{L}\right)^{2}=-L_{M} K / S_{L}$ \\
\hline $\mathrm{LC}$ & $\begin{array}{l}\text { Linear model based on curva- } \\
\text { ture (Markstein 1951) }\end{array}$ & $S_{d}^{*} / S_{L}=1-L_{M} \kappa_{m}$ \\
\hline $\mathrm{NE}$ & $\begin{array}{l}\text { Non-linear model in expan- } \\
\text { sion form (Kelley et al. } \\
\text { 2011) }\end{array}$ & $S_{d}^{*} / S_{L}\left[1+L_{M} \kappa_{m}+L_{M}^{2} \kappa_{m}^{2}+2 / 3 L_{M}^{3} \kappa_{m}^{3}+O\left(L_{M}^{4} \kappa_{m}^{4}\right)\right]=1.0$ \\
\hline $\mathrm{N} 3 \mathrm{P}$ & $\begin{array}{l}\text { Three term extrapolation (Wu } \\
\text { et al. 2005) }\end{array}$ & $S_{d}^{*} / S_{L}=1-L_{M} \kappa_{m}+C \kappa_{m}^{2} \delta_{t h}^{2}$ \\
\hline
\end{tabular}


numbers, but also is more successful in capturing the stretch rate dependence of $S_{d}^{*}$ than the LS model (Kelley and Law 2009; Chen 2011).

Similar to the LS expression, a linear relation between flame speed and flame front curvature was originally proposed by Markstein (1951) based on an empirical assumption involving a Markstein diffusivity $D_{M}=S_{L} L_{M}$ and later observed experimentally (Karpov et al. 1997; Lipatnikov et al. 2015). This relation is referred to as the LC extrapolation in this analysis. The LC extrapolation was subsequently used by Frankel and Sivashinsky (1983) to analyse spherically expanding flames with thermal expansion under the premise of large flame radii. It was found that both NQ and LC extrapolations provide similar results for negative Markstein diffusivity in the case of thermo-diffusively unstable flames, but significant differences have been reported for positive Markstein diffusivities. The LC extrapolation was subsequently extended by Kelley et al. (2011) by including second and third order series contributions of curvature $\kappa_{m}$, which will be referred to as the non-linear equation (NE) extrapolation in this paper. The evaluation of the NE model requires a non-zero value of the term in brackets. The curvature range that ensures a positive value is quite large for the cases considered in this work such that the singularity can effectively be avoided. An alternative variant of the nonlinear extrapolation was suggested by $\mathrm{Wu}$ et al. (2005) where a parameter $C$ associated with a $\kappa_{m}^{2}$ contribution is considered and this non-linear extrapolation with 3 terms will be referred to as the N3P extrapolation in this paper. While the non-linear extrapolation relations offer the potential to better represent the data, this advantage comes at the cost of additional tuning parameters. All the extrapolation relations discussed above and listed in Table 1 were originally proposed for weakly stretched laminar flames. These expressions are often used to extract the unstrained laminar burning velocity from the experimentally obtained flame propagation measurements (Wu and Law 1984; Kelley and Law 2009; Chen 2011; Kelley et al. 2011; Wu et al. 2005). It is common that the stretched laminar flame speed is determined as a function of the unstretched laminar flame speed $S_{L}$ and the stretch rate. Most of the stretch extrapolations shown in Table 1 represent such an explicit "forward" functional relationship, which is a common practice in the context of Reynolds Averaged Navier-Stokes (RANS) / Large Eddy Simulations (LES). This motivates to assess the validity of the extrapolation relations for a range of turbulence intensities $u^{\prime} / S_{L}$ (where $u^{\prime}$ is the root-mean-square (rms) velocity fluctuation), which is yet to be reported in the existing literature. Furthermore, Clavin (1985) indicated that the Markstein length/diffusivity behaviour may change depending on the reaction progress variable $c$ value within the flame and thus it is necessary to assess the sensitivity of $L_{M}$ on $u^{\prime} / S_{L}$ and also on the choice of the value of reaction progress variable $c$ within the flame front. This becomes particularly challenging in the presence of detailed chemistry and transport because the definition of $c$ is not unique and can be done in a number of different ways, which also suggests that the statistics of $S_{d}^{*}$ (and therefore for $L_{M}$ ) are likely to be affected by the choice of $c$. While the Markstein length is rigorously defined as a derivative of a laminar flame speed with respect to the flame stretch rate at the limit of vanishing stretch rate, the terminology is used widely and more generally in the context of turbulent premixed combustion (Brequigny et al. 2016; Venkateswaran et al. 2015; Chaudhuri et al. 2013) and the same approach has been adopted in this work. In this respect, the main objectives of this paper are:

(a) To assess the validity of the extrapolation relations for different values of $u^{\prime} / S_{L}$ across different regimes of premixed turbulent combustion. 
(b) To analyse the sensitivity of the values of $L_{M}$ to the value of $c$ and its definition for different regimes of premixed combustion.

(c) To identify the extrapolation relation which provides the best possible approximation of the local curvature and stretch rate dependences of $S_{d}^{*}$.

The authors recently addressed some of these issues in the context of simple chemistry (Herbert et al. 2020). However, the choice of reaction progress variable on the performance of extrapolation relations could not be addressed there. Hence, this analysis extends these results in the context of detailed chemistry and transport. Thus, the assessment of the extrapolation relations is the main focus of this analysis and the authors are not aware of any study in the existing literature where the aforementioned exercise was undertaken, and the novelty of this work lies in this respect.

In order to meet these objectives, a detailed chemistry DNS database of statistically planar $\mathrm{H}_{2}-$ air premixed flames of equivalence ratio 0.7 (i.e. $\phi=0.7$ ) spanning different regimes of premixed turbulent combustion has been considered. The choice of $\phi=0.7$ is driven by the fact that $\mathrm{H}_{2}-$ air premixed flames are nominally thermo-diffusively neutral with respect to the stretch effects on $S_{d}^{*}$ at this equivalence ratio (Chen and Im 1998; Im and Chen 2002). However, different species have different Lewis number even through the flame might be nominally thermo-diffusively neutral, which necessitates the assessment of the performance of the extrapolation relations for different species with varying Lewis numbers $L e$, including major species with $L e \ll 1$ (e.g. $\mathrm{H}_{2}$ ). In order to address this aspect, the aforementioned detailed chemistry DNS database has been utilised to assess the performance of the LS, NQ, LC, NE and N3P extrapolations for multiple values of the progress variable $c$ across the flame for different definitions of reaction progress variable.

The rest of the paper is organised as follows. The mathematical background and numerical implementation pertaining to this analysis are discussed in the next two sections. Following that, results will be presented and subsequently discussed before the conclusions are drawn.

\section{Mathematical background}

The reaction progress variable $c$ is defined (Bray 1980) based on a suitable reactant or product mass fraction $Y_{R}$ as:

$$
c=\left(Y_{R 0}-Y_{R}\right) /\left(Y_{R 0}-Y_{R \infty}\right)
$$

where $Y_{R}$ is the mass fraction of the reactant which is used for the definition of reaction progress variable. In this analysis, reaction progress variables based on $\mathrm{H}_{2}, \mathrm{O}_{2}$ and $\mathrm{H}_{2} \mathrm{O}$ mass fractions are considered. The differential diffusion between heat and mass due to the presence of light species in $\mathrm{H}_{2}$-air premixed flames may give rise to local occurrences of $\mathrm{H}_{2} \mathrm{O}$ mass fractions higher than the maximal values found in laminar flame data. Indeed, such behaviour can be locally observed but does not affect the current analysis where reaction progress variable values of $0.3,0.5,0.8$ are primarily considered because the peak value of reaction progress variable reaction rate takes place within this region in laminar $\mathrm{H}_{2}$-air flames with $\phi=0.7$ (Klein et al. 2018). Figure 1 shows the reaction rate magnitude $\dot{\omega}_{Y}$ normalised by the maximum absolute value of reaction rate together with the reaction progress variable $c_{Y}$ for major species $\mathrm{Y}=\mathrm{H}_{2}, \mathrm{H}_{2} \mathrm{O}, \mathrm{O}_{2}$ versus non-dimensional temperature $\theta=\left(T-T_{0}\right) /\left(T_{a d}-T_{0}\right)$ (with $T, T_{0}$ and $T_{a d}$ being the instantaneous temperature, 
Fig. 1 Magnitude of the reaction rate $\dot{\omega}_{Y}$ normalised with the maximum absolute value of reaction rate (solid lines) and reaction progress variable $c_{Y}$ for major species $Y=\mathrm{H}_{2}, \mathrm{H}_{2} \mathrm{O}, \mathrm{O}_{2}$ (dashed lines) versus non-dimensional temperature $\theta$ based on temperature for laminar flame data

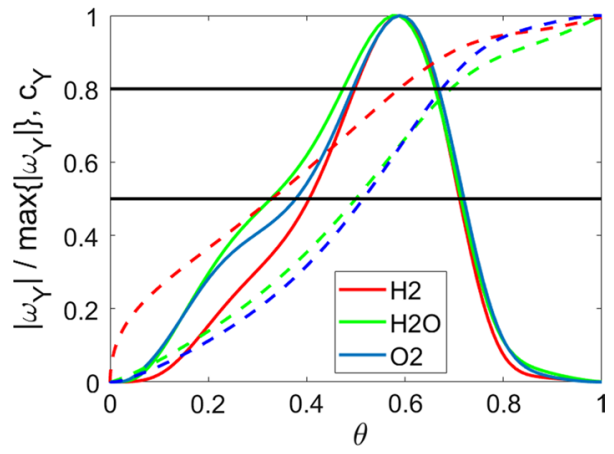

unburned gas temperature and adiabatic flame temperature, respectively) based on laminar $\mathrm{H}_{2}$ - air flame data with $\phi=0.7$. Figure 1 indicates that this peak location corresponds approximately to $c_{\mathrm{H}_{2}} \approx 0.8, c_{\mathrm{H}_{2} \mathrm{O}} \approx 0.6, c_{\mathrm{O}_{2}} \approx 0.6$. This demonstrates that the choice of reaction progress variable values selected for this analysis covers the relevant range of $c$ values for which the results are presented in Sect. 4 and roughly corresponds to the location of maximum reaction rates.

The transport equation of $c$ is given by (Chakraborty and Cant 2011; Sabelnikov et al. 2017; Peters 2000; Pope 1988; Hawkes and Cant 2001; Chakraborty and Cant 2009; Bray 1980):

$$
\rho[\partial c / \partial t+\vec{u} \cdot \nabla c]=\dot{w}+\nabla \cdot\left(\rho D_{c} \nabla c\right)
$$

where $\vec{u}$ is the fluid velocity and $\dot{w}=-\dot{w}_{Y} /\left(Y_{R 0}-Y_{R \infty}\right)$ with $\dot{w}_{Y}$ being the net reaction rate of the corresponding species. In the context of mixture-averaged transport, as employed in the present work, the reaction progress diffusivity $D_{c}$ of a species is determined by $D_{c}=\left(1-Y_{k}\right) / \sum_{j \neq k} X_{j} / D_{j k}$ where $X_{j}$ is the mole fraction of species $j, D_{j k}$ is the binary diffusion coefficient, and species $k$ is used to define the reaction progress variable (where $k \in\left\{\mathrm{H}_{2}, \mathrm{O}_{2}, \mathrm{H}_{2} \mathrm{O}\right\}$ has been used for the analysis). The thermo-physical properties used for the DNS computations are used for the analysis of this paper. The kinematic form of the transport equation of a given $c$ isosurfaces can be written as (Chakraborty and Cant 2011, 2009; Sabelnikov et al. 2017; Peters 2000; Pope 1988; Hawkes and Cant 2001):

$$
\rho[\partial c / \partial t+\vec{u} \cdot \nabla c]=\rho_{0} S_{d}^{*}|\nabla c|
$$

A comparison of Eqs. 2 and 3 indicates that:

$$
S_{d}^{*}=\left[\dot{w}+\nabla \cdot\left(\rho D_{c} \nabla c\right)\right] /\left(\rho_{0}|\nabla c|\right)
$$

Equation 4 has been utilised to obtain displacement speed from DNS data, and thus the choice of the mass fraction for the definition of $c$ affects the statistics of $S_{d}^{*}$. Using the definition of $S_{d}^{*}$, one gets the following expression for the FSD based reaction rate closure (Chakraborty and Cant 2011; Boger et al. 1998):

$$
\overline{\left[\dot{w}+\nabla \cdot\left(\rho D_{c} \nabla c\right)\right]}=\overline{\left(\rho_{0} S_{d}^{*}\right)} \Sigma_{s}
$$

where $\bar{Q}$ and $\overline{(Q)_{s}}=\overline{Q|\nabla c|} / \Sigma_{\text {gen }}$ are the Reynolds averaged/LES filtered and surfaceweighted average/filtered value of a general quantity $Q$, respectively with $\Sigma_{\text {gen }}=\overline{|\nabla c|}$ 
being the generalised FSD (Chakraborty and Cant 2011; Boger et al. 1998). Equation 5 shows that it is not sufficient to model the generalised FSD $\Sigma_{\text {gen }}$ but a robust extrapolation

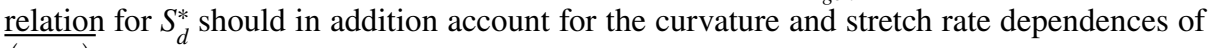
$\left(\rho_{0} S_{d}^{*}\right)_{s}$ with sufficient accuracy (Hawkes and Cant 2001; Boger et al. 1998). This is important because the assumption is not valid in turbulent flames, as demonstrated in several previous analyses (Chakraborty and Cant 2011, 2009; Sabelnikov et al. 2017). It is worth mentioning that the modelling of $\overline{\left(\rho S_{d}\right)}=\rho_{0} \overline{\left(S_{d}^{*}\right)}$ is closely linked to the estimation of the stretch factor $I_{0}$, in the expression $\overline{\left(\rho S_{d}\right)_{s}}=I_{0} \rho_{0} S_{L}$ introduced by Bray (1980) because $I_{0}$ can be expressed as: $I_{0}=\overline{\left(S_{d}^{*}\right)} / S_{L}$.

The extrapolation relations in Table 1 involve flame curvature $\kappa_{m}$ and stretch rate $K$, which are defined as (Pope 1988):

$$
\kappa_{m}=0.5 \nabla \cdot \vec{N} \text { and } K=\nabla \cdot \vec{u}-(\vec{N} \cdot \nabla \vec{u}) \cdot \vec{N}+2 S_{d} \kappa_{m}
$$

where $\vec{N}=-\nabla c /|\nabla c|$ is the flame normal vector which points towards the reactants and according to this convention a positive curvature is associated with a flame surface element, which is convex towards the reactants.

The performances of the extrapolation relations have been quantified in terms of the Pearson correlation coefficient between $S_{d}^{*}$ predicted by the extrapolation relations and the same quantity extracted from the DNS data. The Pearson correlation coefficient, which measures the linear dependence between two variables $A$ and $B$, is given by Ahlgren et al. (2003):

$$
r(A, B)=\operatorname{cov}(A, B) / \sigma_{A} \sigma_{B}
$$

where $\sigma_{\mathrm{A}}$ and $\sigma_{\mathrm{B}}$ are the standard deviations of $A$ and $B$, respectively and $\operatorname{cov}(A, B)$ is the co-variance of random variables $A$ and $B$.

\section{Numerical implementation}

A skeletal chemical mechanism (Burke et al. 2012) with 9 species and 19 chemical reactions has been utilised to develop a three-dimensional DNS (Im et al. 2016; Wacks et al. 2016; Papapostolou et al. 2017) database of $\mathrm{H}_{2}$-air flames with an equivalence ratio of 0.7. An atmospheric combustion with unburned gas temperature $T_{0}=300 \mathrm{~K}$ is considered, which gives rise to an unstrained laminar burning velocity $S_{L}=135.6 \mathrm{~cm} / \mathrm{s}$ and heat release parameter $\tau=\left(T_{a d}-T_{0}\right) / T_{0}=5.71$. The information related to numerical implementation of this database has been discussed in detail elsewhere (Im et al. 2016; Wacks et al. 2016; Papapostolou et al. 2017), and thus will not be repeated here. Turbulent inflow and outflow boundaries are specified in the direction of mean flame propagation and transverse boundaries are considered to be periodic. An improved version of the Navier Stokes characteristic boundary conditions (NSCBC) technique (Yoo et al. 2005) is used to specify the inflow and outflow boundaries. The turbulent velocity fluctuations at the inflow are specified by scanning a plane through a frozen homogeneous isotropic incompressible velocity fluctuation field generated using a pseudo-spectral method (Rogallo 1981) following the Passot-Pouquet spectrum (Passot and Pouquet 1987). The temporal evolution of flame area has been monitored and the simulations are performed until the flame area no longer varies with time and the flame is considered to be statistically stationary. The inflow values of normalised root-mean-square turbulent velocity fluctuation $u^{\prime} / S_{L}$, 
Table 2 List of inflow turbulence parameters

\begin{tabular}{lccccc}
\hline Case & $u^{\prime} / S_{L}$ & $l_{T} / \delta_{\text {th }}$ & $\mathrm{Re}_{t}$ & $D a$ & $K a$ \\
\hline A & 0.7 & 14.0 & 227 & 20.0 & 0.75 \\
B & 5.0 & 14.0 & 1623 & 2.8 & 14.4 \\
C & 14.0 & 4.0 & 1298 & 0.29 & 126 \\
\hline
\end{tabular}

turbulent length scale to flame thickness ratio $l_{T} / \delta_{t h}$, Damköhler number $D a=l_{T} \mathrm{~S}_{L} / u^{\prime} \delta_{t h}$, Karlovitz number $K a=\left(\rho_{0} S_{L} \delta_{t h} / \mu_{0}\right)^{0.5}\left(u^{\prime} / S_{L}\right)^{1.5}\left(l_{T} / \delta_{t h}\right)^{-0.5}$ (expressed here as the ratio of chemical $\delta_{t h} / S_{L}$ to Kolmogorov timescale $\left.\left(\mu_{0} l / \rho_{0} u^{\prime 3}\right)^{0.5}\right)$ and turbulent Reynolds number $R e_{t}=\rho_{0} u^{\prime} l_{T} / \mu_{0}$ for all cases are listed in Table 2 where $\mu_{0}$ is the unburned gas viscosity, $\delta_{t h}=\left(T_{a d}-T_{0}\right) / \max |\nabla T|_{L}$ is the thermal flame thickness and the subscript ' $\mathrm{L}$ ' is used to refer to unstrained laminar flame quantities. Cases A-C represent the corrugated flamelets $(K a<1)$, thin reaction zones $(1<K a<100)$ and broken reaction zones $(K a>100)$ regimes (Peters 2000) of premixed combustion, respectively according to the regime diagram by Peters (Peters 2000).

The domain size is $20 \mathrm{~mm} \times 10 \mathrm{~mm} \times 10 \mathrm{~mm}(8 \mathrm{~mm} \times 2 \mathrm{~mm} \times 2 \mathrm{~mm})$ in cases $\mathrm{A}$ and B (case C), which has been discretised by a uniform Cartesian grid of $512 \times 256 \times 256$ $(1280 \times 320 \times 320)$. Simulations have been carried out for $1.0 t_{e}, 6.8 t_{e}$ and $6.7 t_{e}$ (i.e. $t_{e}=l_{T} / u^{\prime}$ ) for cases A-C respectively, and this simulation time remains comparable to several previous analyses (Hun and Huh 2008; Reddy and Abraham 2012; Pera et al. 2013; Dopazo et al. 2015). In this regard, it is noted that the longitudinal integral scale $L_{11}$ is a factor of 2.5 smaller than the most energetic scale $l_{T}$ for cases A-C. Consequently, the values of Karlovitz (Damköhler) number in Table 1 will increase (decrease) by a factor 1.6 (2.5) if $L_{11}$ instead of $l_{T}$ is used for their definitions. Therefore, the run-time in terms of the initial eddy-turn over time $L_{11} / u^{\prime}$ is at least 2.5 times that of $t_{e}=l_{T} / u^{\prime}$ and thus the runtime is $\{2.5,17,16.75\} L_{11} / u^{\prime}$ for cases A-C, respectively.

\section{Results and discussion}

The scatters of the normalised density-weighted displacement speed $S_{d}^{*} / S_{L}$ in response to the variations of normalised curvature $\kappa_{m} \times \delta_{t h}$ for $c=0.3,0.5$ and 0.8 isosurfaces for cases A-C for reaction progress variable definition based on $\mathrm{H}_{2}$ mass fraction are exemplarily shown in Fig. 2. The corresponding normalised stretch rate $K \times \delta_{t h} / S_{L}$ dependence of the normalised density-weighted displacement speed $S_{d}^{*} / S_{L}$ are shown in Fig. 3. The corresponding variations for the reaction progress variable definitions based on $\mathrm{O}_{2}$ and $\mathrm{H}_{2} \mathrm{O}$ mass fractions are qualitatively similar to those shown in Figs. 2, 3 and thus are not explicitly shown here for the sake of conciseness. The maximum reaction rates for these major product or reactant species $\left(\mathrm{H}_{2}, \mathrm{O}_{2}, \mathrm{H}_{2} \mathrm{O}\right)$ are (for laminar flame data) found at a value of $\theta \approx 0.6$ (see Fig. 1). Figure 1 shows that the peak location corresponds approximately to reaction progress variable values based on $\mathrm{H}_{2}, \mathrm{O}_{2}$ and of $\mathrm{H}_{2} \mathrm{O}$. This demonstrates that the choice of reaction progress variable values selected for this analysis covers the range of $c$ where the peak reaction rate occurs. The mean values of $S_{d}^{*} / S_{L}$ conditioned upon the $\kappa_{m} \delta_{t h}$ and $K \delta_{t h} / S_{L}$ values according to the predictions of the extrapolation relations in Table 1 are also shown in Figs. 2, 3, respectively. In Figs. 2, 3, for the extrapolation relations the parameters $L_{M}$ and $C$ have been estimated based on a non-linear regression analysis. The parameter $L_{M}$ is fitted to the datapoints in Fig. 2 for the extrapolation relations which 


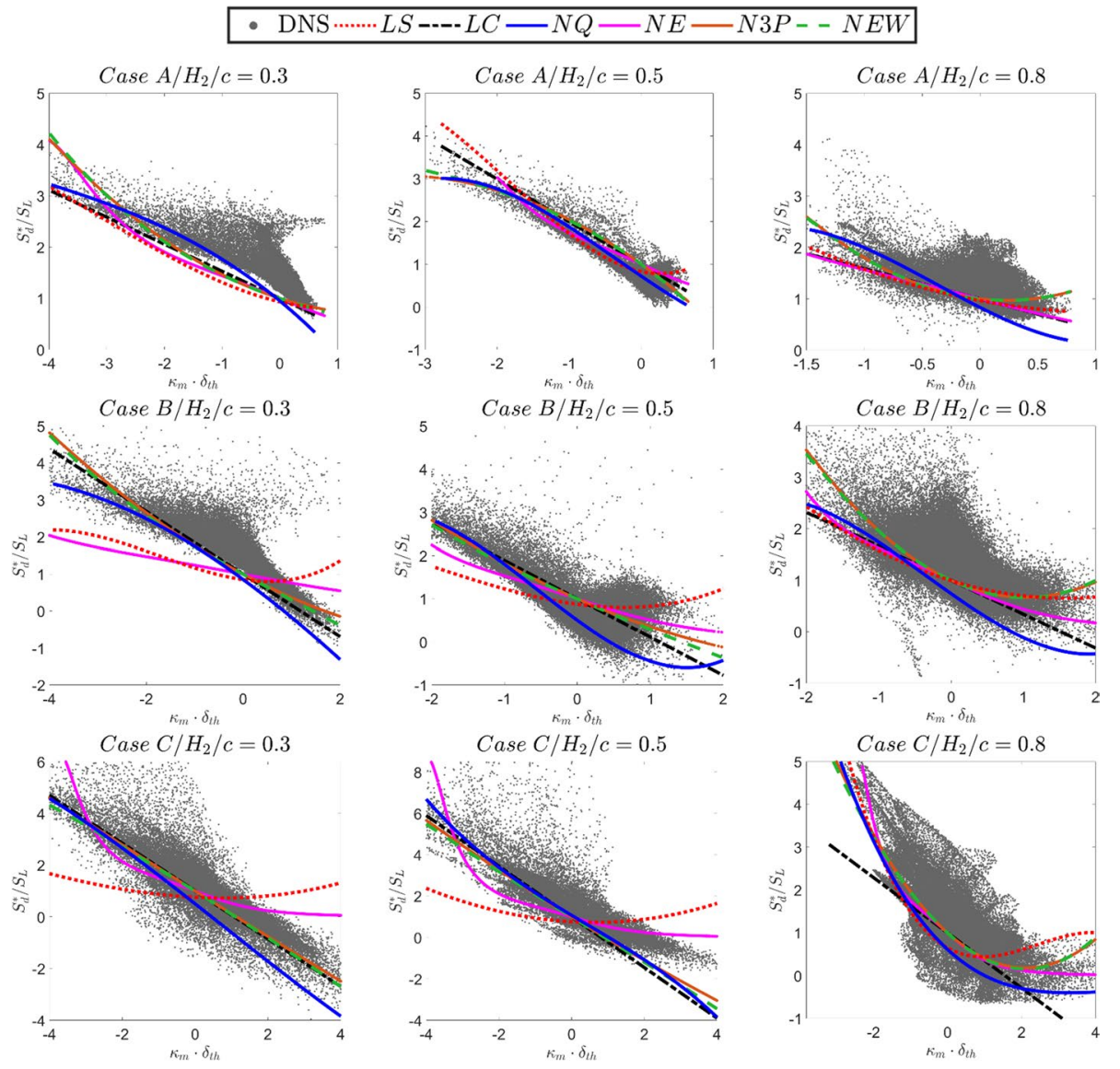

Fig. 2 Scatter of $S_{d}^{*} / S_{L}$ with $\kappa_{m} \delta_{t h}$ along with the mean values of $S_{d}^{*} / S_{L}$ conditioned upon $\kappa_{m} \delta_{t h}$ according to LS, LC, NQ, NE, N3P and NEW extrapolations on $c=0.3,0.5$ and 0.8 isosurfaces for cases A-C ( $1 \mathrm{st}-$ 3rd rows) for reaction progress variable definitions based on $\mathrm{H}_{2}$, mass fraction. Note that N3P and NEW extrapolations fall almost upon each other in Figs. 2 and 3

expresses $S_{d}^{*}$ as a function of $\kappa_{m}$ not those in Fig. 3 (i.e. it is fitted as a function of mean curvature rather than stretch). Similarly, $L_{M}$ is fitted to the datapoints in Fig. 3 for the LS extrapolation and not to those in Fig. 2. Figures 2, 3 reveal that the LC and LS extrapolations do not adequately capture the non-linear stretch rate dependence of $S_{d}^{*} / S_{L}$ for all cases irrespective of the choice of reaction progress variable definition and its value. In contrast, the LC relation shows a reasonable curvature dependence of $S_{d}^{*} / S_{L}$ and this holds especially true for case $\mathrm{C}$ where the influence of stretch rate is diminished, possibly because of the relatively high frequency of velocity fluctuations for this case. This can be substantiated from the correlation coefficients between $S_{d}^{*} / S_{L}$ and $\kappa_{m} \times \delta_{t h}$, and between $S_{d}^{*} / S_{L}$ and $K \times \delta_{t h} / S_{L}$ for $c=0.3,0.5$ and 0.8 isosurfaces for cases A-C for reaction progress variable definitions based on $\mathrm{H}_{2}, \mathrm{O}_{2}$ and $\mathrm{H}_{2} \mathrm{O}$ mass fractions in Table 3 .

A note of caution is that the correlation coefficient is invariant to constant multipliers and therefore the results of the correlation analysis cannot be directly compared to Figs. 2, 3 . The negative correlation between $S_{d}^{*}$ and $\kappa_{m}$ with a correlation coefficient different from 


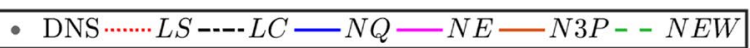
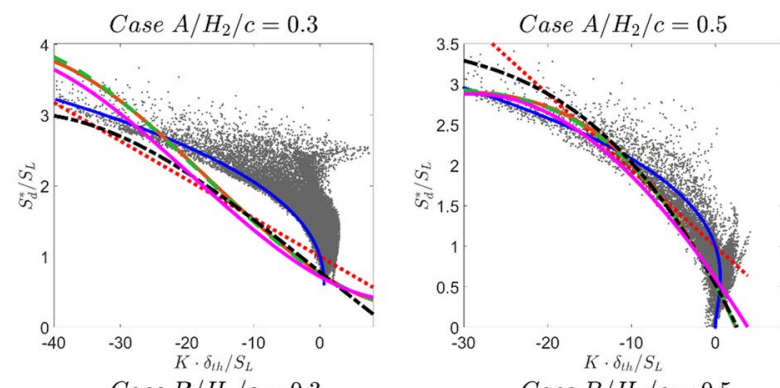

Case $B / H_{2} / c=0.5$
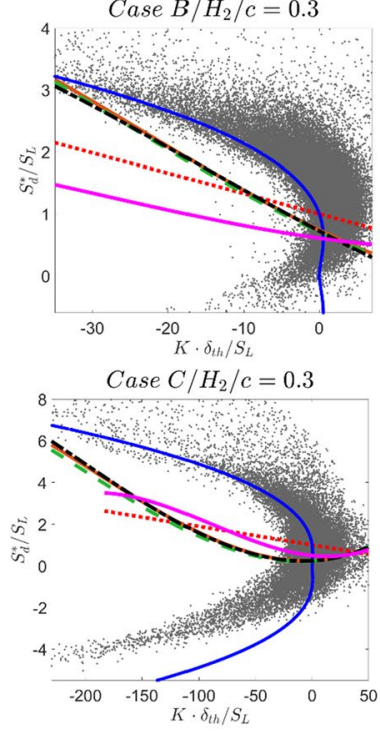

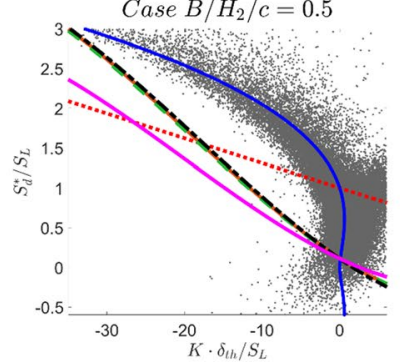

Case $\mathrm{C} / \mathrm{H}_{2} / \mathrm{c}=0.5$

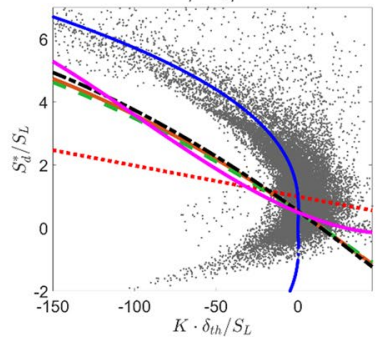

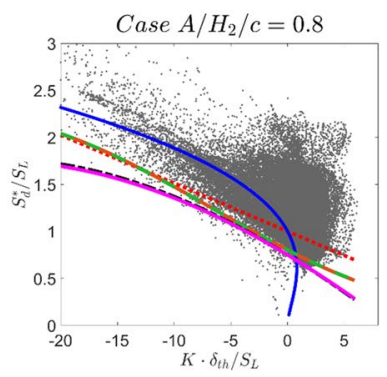

Case $B / H_{2} / c=0.8$

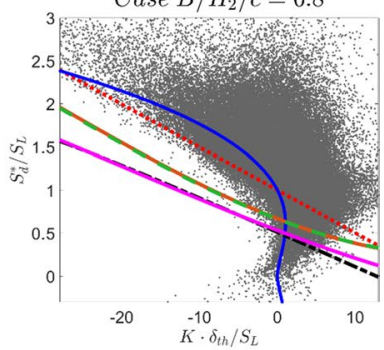

Case $\mathrm{C} / \mathrm{H}_{2} / \mathrm{c}=0.8$

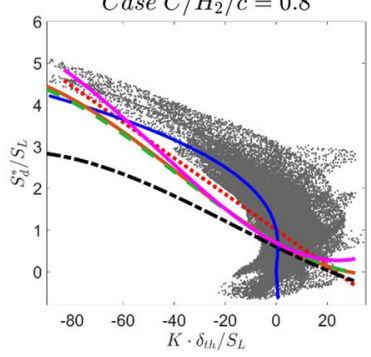

Fig. 3 Scatter of $S_{d}^{*} / S_{L}$ with $K \delta_{t h} / S_{L}$ along with the mean values of $S_{d}^{*} / S_{L}$ conditioned upon $K \delta_{t h} / S_{L}$ according to LS, LC, NQ, NE, N3P and NEW extrapolations on $c=0.3,0.5$ and 0.8 isosurfaces for cases A-C (1st-3rd rows) for reaction progress variable definitions based on $\mathrm{H}_{2}$ mass fraction

Table 3 Correlation coefficients between $S_{d}^{*}$ and $\kappa_{m}$, and between $S_{d}^{*}$ and $K$ for different values of $c$ for different definitions of reaction progress variable

\begin{tabular}{|c|c|c|c|c|c|c|}
\hline \multirow[b]{2}{*}{$c /$ Species } & \multicolumn{2}{|l|}{ Case A } & \multicolumn{2}{|l|}{ Case B } & \multicolumn{2}{|l|}{ Case C } \\
\hline & $S_{d}^{*}-K$ & $\overline{S_{d}^{*}-\kappa_{m}}$ & $d-K$ & ${ }_{d}^{*}-\kappa_{m}$ & $S_{d}^{*}-K$ & $\overline{S_{d}^{*}-\kappa_{m}}$ \\
\hline $\mathbf{0 . 3 / \mathbf { H } _ { 2 }}$ & -0.727 & -0.796 & -0.763 & -0.031 & -0.596 & \\
\hline $0.5 / \mathbf{H}_{2}$ & -0.869 & -0.918 & -0.701 & -0805 & -0.745 & -0.903 \\
\hline $0.8 / \mathbf{H}_{2}$ & -0.509 & -0.518 & -0.652 & -0.040 & -0.654 & -0.711 \\
\hline $0.3 / \mathrm{H}_{2} \mathrm{O}$ & -0.904 & -0.925 & -0.820 & -0.928 & -0.601 & -0.871 \\
\hline $0.5 / \mathrm{H}_{2} \mathrm{O}$ & & & & -0.7 & & -0.863 \\
\hline $0.8 / \mathrm{H}_{2} \mathrm{O}$ & -0.705 & -0.570 & -0.805 & -0.643 & -0.593 & -0.686 \\
\hline $0.3 / \mathrm{O}_{2}$ & -0.927 & -0.924 & -0.851 & -0.907 & -0.254 & -0.870 \\
\hline & -0.857 & -0.787 & -0.839 & -0.799 & -0.348 & -0.805 \\
\hline $0.8 / \mathrm{O}_{2}$ & -0.664 & -0.496 & -0.774 & -0.546 & -0.568 & -0.641 \\
\hline
\end{tabular}


-1.0 indicates a negative, nonlinear correlation and is consistent with several previous DNS findings (Chen and Im 1998; Chakraborty et al. 2007, 2011a,b; Venkateswaran et al. 2015; Hun and Huh 2008; Echekki and Chen 1996; Peters et al. 1998; Chakraborty and Cant 2004, 2005; Chakraborty 2007). It can be seen from Figs. 2, 3 that NE and N3P extrapolations qualitatively predict the non-linear $\kappa_{m}$ dependences of $S_{d}^{*}$ when the optimum values of the model parameters (i.e. normalised Markstein length $L_{M} / \delta_{t h}$ and the model parameter $C$ in the N3P extrapolation) are used because of their functional forms (see Table 1) but locally there are discrepancies between the quantitative agreements between DNS data and the predictions of the extrapolation relations. However, the predictions of NE and N3P extrapolations differ from each other for large curvature magnitudes for both positive and negative $\kappa_{m}$ values. On the other hand, the non-linear NQ extrapolation captures the qualitative nature of the stretch rate $K$ dependence of $S_{d}^{*}$, while both NE and N3P extrapolations do not adequately predict the interrelation between $K$ and $S_{d}^{*}$. Similarly, the predictions of the curvature $\kappa_{m}$ dependences of $S_{d}^{*}$ by the NQ extrapolation are qualitatively different from that obtained from NE and N3P extrapolations. It can be seen from Fig. 3 that the LC extrapolation allows for some degree of non-linearity in $K$ dependences of $S_{d}^{*}$ but it does not capture the qualitative nature of stretch rate dependence of density-weighted displacement speed.

It is useful to consider different components of density-weighted displacement speed (Chen and Im 1998; Chakraborty et al. 2007, 2011a, b; Venkateswaran et al. 2015; Hun and Huh 2008; Echekki and Chen 1996; Peters et al. 1998; Chakraborty and Cant 2004, 2005; Chakraborty 2007) in order to explain the observations made from Figs. 2, 3 as:

$$
S_{r}^{*}=\dot{w} / \rho_{0}|\nabla c| ; S_{n}^{*}=\vec{N} \cdot \nabla\left(\rho D_{c} \vec{N} \cdot \nabla \mathrm{c}\right) / \rho_{0}|\nabla c| ; S_{t}^{*}=-2 \rho D_{c} \kappa_{m} / \rho_{0}
$$

where $S_{r}^{*}, S_{n}^{*}$ and $S_{t}^{*}$ are the reaction component, normal diffusion component and tangential diffusion components. It can be seen from Eq. 8 that $S_{t}^{*}$ is linearly related to $\kappa_{m}$, and Eq. 8 can be utilised to demonstrate that the stretch rate can be expressed as: $K=a_{T}+2\left(\rho_{0} / \rho\right) S_{d}^{*} \kappa_{m}=a_{T}+2\left(\rho_{0} / \rho\right)\left(S_{r}^{*}+S_{n}^{*}\right) \kappa_{m}-4 D_{c} \kappa_{m}^{2}$. It can be seen from Table 3 that $S_{d}^{*}$ is mostly strongly negatively correlated with curvature, which reveals that the curvature stretch is likely to induce a significant non-linear curvature dependence of $K$. For thermo-diffusively neutral flames $\left(S_{r}^{*}+S_{n}^{*}\right)$ remains weakly correlated with curvature $\kappa_{m}$ (Chakraborty and Cant 2004, 2005; Chakraborty 2007; Chakraborty et al. 2011a, 2011b) and thus the correlation coefficient between $S_{d}^{*}$ and $\kappa_{m}$ remain mostly close to -1.0 (note a correlation coefficient of -1.0 is indicative of a linear relation) except for $c=0.8$ for $\mathrm{O}_{2}$ based reaction progress variable (see Table 3 ). This suggests that a non-linear stretch rate $\mathrm{K}$ dependence of $S_{d}^{*}$ is expected, as shown in Fig. 3. Furthermore, an assumption of the linear extrapolation (i.e. LS extrapolation) leads to the following expression of $L_{M}$ (Chen and Im 1998; Chakraborty et al. 2007):

$$
L_{M}=\left[S_{L}-S_{d}^{*}\right] /\left[a_{T}+2 S_{d} \kappa_{m}\right]
$$

It is worth noting that Eq. 9 suffers from a singularity for $K=0$ and it cannot be used under this condition. However, under turbulent conditions, $K \neq 0$ in most locations. Moreover $L_{M}$, has no relevance for $K=0$ because under that condition one gets: $S_{d}^{*}=S_{L}$. When $K \neq 0$, it can be concluded from Eq. 9 that $L_{M}$ is expected to scale as: $L_{M} \sim\left(-1 / 2 \kappa_{m}\right)$ for $\left|2 S_{d} \kappa_{m}\right| \gg a_{T}$, and thus a constant value of $L_{M}$ in the context of LS extrapolation may not be sufficient to represent $S_{d}^{*}$ behaviour. Equation 9 also suggests that it is possible to have 


\section{$L S \square N Q \square L C \square N E \square N 3 P \square C$}
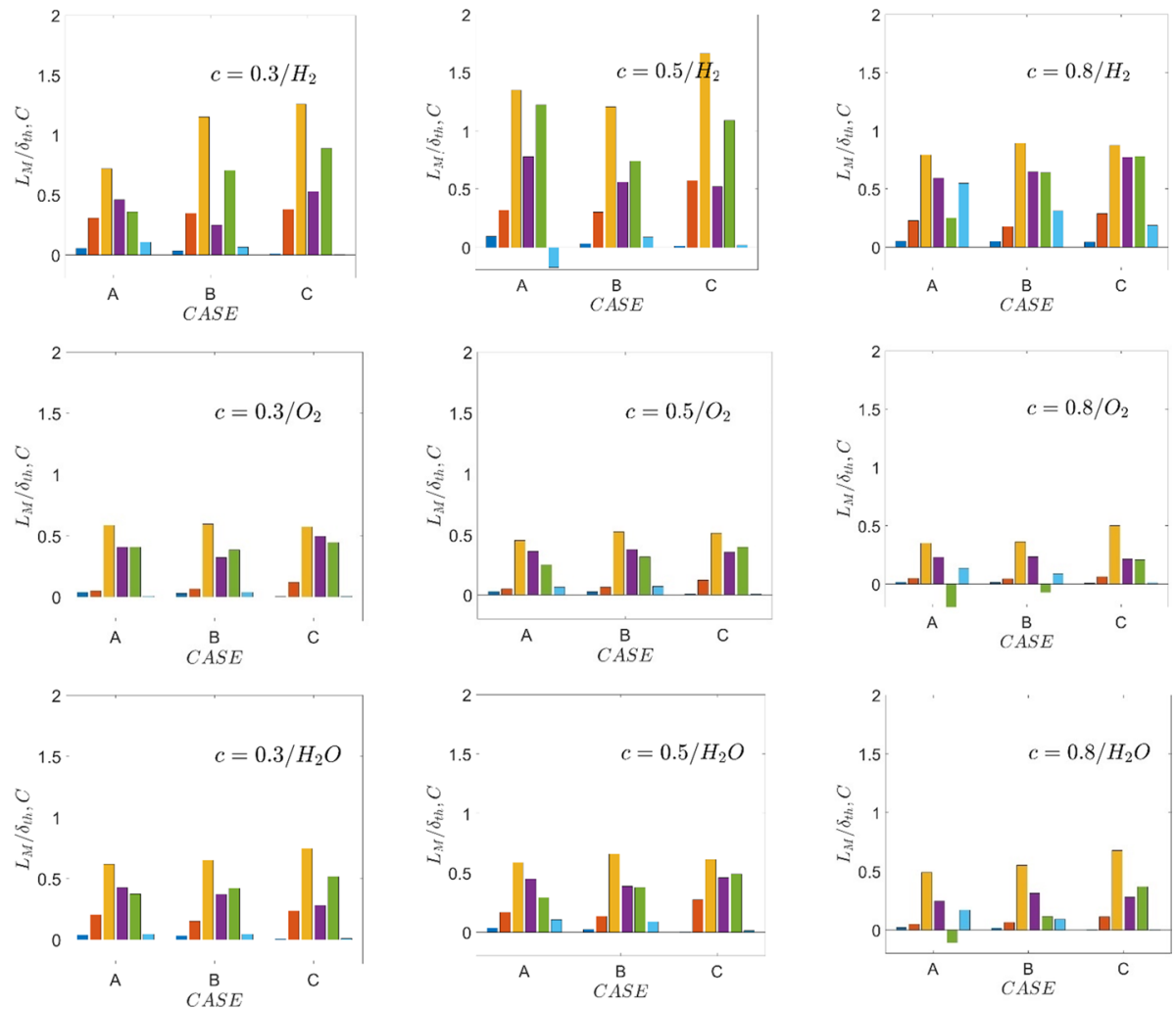

Fig. 4 Variations of the optimised values of $L_{M} / \delta_{t h}$ and $C$ for $c=0.3,0.5$ and 0.8 isosurfaces (1st-3rd column) in cases A-C for reaction progress variable definitions based on $\mathrm{H}_{2}, \mathrm{O}_{2}$ and $\mathrm{H}_{2} \mathrm{O}$ mass fractions (1st-3rd row)

negative Markstein length for large positive curvature locations for the non-zero values of stretch rate $K$.

The variations of the optimised normalised Markstein length $L_{M} / \delta_{t h}$ and the model parameter $C$ in the N3P extrapolation based on a regression analysis are shown in Fig. 4 for $c=0.3$, 0.5 and 0.8 isosurfaces for cases A-C for different choices of reaction progress variable. Figure 4 shows that $L_{M} / \delta_{t h}$ for all the extrapolation relations remains of the order of unity (i.e. $\left.L_{M} / \delta_{t h} \sim O(1)\right)$ except for the LS extrapolation for all values of $c$ in all cases. The $L_{M} / \delta_{t h}$ values for the LC extrapolation have been found to be higher for $\mathrm{H}_{2}$ mass fraction based reaction progress variable than in the cases with $\mathrm{O}_{2}$ and $\mathrm{H}_{2} \mathrm{O}$. It can further be seen from Fig. 4 that the LC extrapolation yields the highest value of $L_{M} / \delta_{t h}$ among all the extrapolation relations listed in Table 1, whereas the lowest value of $L_{M} / \delta_{t h}$ is obtained for the LS extrapolation. This behaviour is observed for all values of $c$ irrespective of the choice of reaction progress variable. It can be discerned from Fig. 4 that the $L_{M} / \delta_{t h}$ values do not show any consistent monotonic trend with the change of reaction progress variable $c$ within the flame front. The $L_{M} / \delta_{t h}$ values increase with increasing $K a$ (i.e. from case A to case $\mathrm{C}$ ) for the NQ extrapolation but no monotonic trend has been observed for the Markstein length for other extrapolations. 
It is difficult to identify the exact reasons for differences between different progress variable definitions. However, it is not surprising that there are differences. For simple chemistry, $L_{M}$ for $S_{d}^{*}$ according to the analytical relation can be given by Clavin and Joulin (1983):

$$
L_{M} / \delta_{t h}=(1+\tau) \ln (1+\tau) / \tau+0.5\left(\beta_{Z} / \tau\right)(L e-1) \int_{0}^{\tau}\{\ln (1+x) / x\} d x
$$

where $D_{0}$ is the unburned gas diffusivity, $\tau$ is the heat release parameter $L e$ is the Lewis number and $\beta_{Z}$ the Zel'dovich number (i.e. $\beta_{Z}=T_{a c}\left(T_{a d}-T_{0}\right) / T_{a d}^{2}$ with $T_{a c}$ is the activation temperature). While some parameters cannot be defined for individual species, it is obvious that some other parameters are species-dependent. As an example, the effective or global Lewis number can be substantially different to the species Lewis number, and also the flame thickness $\delta_{L}=1 / \max |\nabla c|_{L}$ of individual species can be substantially different to $\delta_{t h}$.

It is worth mentioning that normalisation of $L_{M}$ by $\delta_{L}$ (with $\delta_{t h} / \delta_{L}=1.03,1.18,1.29$ for the definition of $c$ based on $\mathrm{H}_{2}, \mathrm{H}_{2} \mathrm{O}$ and $\mathrm{O}_{2}$ mass fraction respectively) brings $L_{M} / \delta_{L}$ a little bit closer to unity but does not significantly alter the behaviour shown in the bar charts and thus is not explicitly shown here. The coefficient $C$ for the N3P extrapolation remains small in magnitude in comparison to that of $L_{M} / \delta_{\text {th }}$ for all the cases irrespective of the choice of the definition of reaction progress variable. The value of $C$ remains negative for $c=0.5$ for $\mathrm{H}_{2}$ mass fraction-based reaction progress variable for case A but for all other definitions and values of $c$, and also in other cases, only positive values of $C$ are obtained. The correlation coefficients between $S_{d}^{*}$ obtained from DNS data and the predicted values according to the extrapolation relations using the optimum values of $L_{M} / \delta_{\text {th }}$ and $\mathrm{C}$ (as shown in Fig. 4) are shown in Fig. 5 for different values and definitions of $c$ for cases A-C. The LC, NQ and N3P extrapolations exhibit high correlation coefficient values (i.e. consistently close to unity) with comparable magnitudes for all values and definitions of $c$ for cases $\mathrm{B}$ and $\mathrm{C}$, whereas the correlation coefficients for case A remain smaller than 0.4 for $\mathrm{H}_{2}$ mass fraction based reaction progress variable. However, the correlation coefficients for the LC, NQ and N3P extrapolations remain high and have comparable values for case $\mathrm{A}$ for $\mathrm{O}_{2}$ and $\mathrm{H}_{2} \mathrm{O}$ mass fraction based reaction progress variables. The correlation coefficients for the LS extrapolation decrease with increasing $K a$ (i.e. from case A to case C) and a similar trend is observed for the NE extrapolation towards the unburned gas side of the flame front. The variations of the correlation coefficient for the NE extrapolation in the middle and towards the burned gas side of the flame front are found to be mostly qualitatively similar to that of the LC, NQ and N3P extrapolations but the magnitude of the correlation coefficient for the NE extrapolation remains smaller than the LC, NQ and N3P extrapolations. The correlation coefficient for the LS extrapolation shows the smallest value amongst all the extrapolations considered here for case $C$ irrespective of the value of definition of $c$. Further, on average, over all cases and definitions, the LC extrapolation exhibits higher values of the correlation coefficient than the LS extrapolation. This originates from a non-linear $K$ dependence of $S_{d}^{*}$ according to $K=a_{T}+2\left(\rho_{0} / \rho\right) S_{d}^{*}\left(S_{L}-S_{d}^{*}\right) / L_{M}$ when using the LC extrapolation which is not captured in the case of the LS extrapolation.

A correlation coefficient with a magnitude smaller than 1 implies a non-linear relation. Hence, it is evident from Fig. 5 that the interrelation between $S_{d}^{*}$ and the stretch rate (or curvature) becomes significantly non-linear for high values of $u^{\prime} / S_{L}$ (e.g. see Figs. 2, 3 for case $\mathrm{C}$ ). Because of the non-linear functional form, the NQ, NE and N3P extrapolations perform relatively better for high values of $u^{\prime} / S_{L}$ and $K a$. However, the practical usage of the NQ extrapolation requires solution of a non-linear equation (see Table 1) and this 

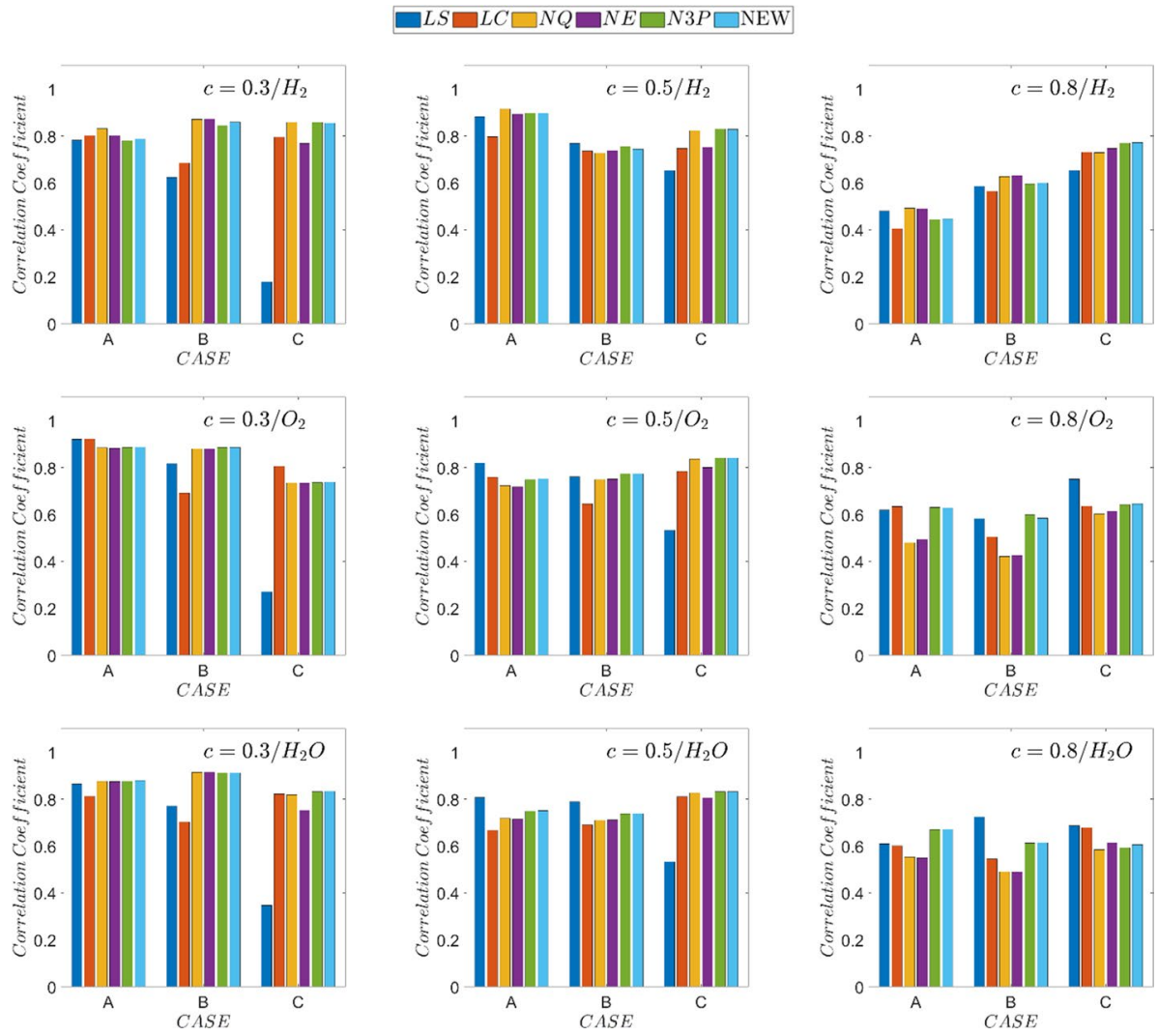

Fig. 5 Variations of correlation coefficients between $S_{d}^{*}$ obtained from DNS data and the predicted values according to the extrapolation relations using the optimum values of $L_{M} / \delta_{t h}$ and $C$ (as shown in Fig. 3) for $c=0.3,0.5$ and 0.8 isosurfaces ( $1 \mathrm{st}-3 \mathrm{rd}$ column) in cases $\mathrm{A}-\mathrm{C}$ for reaction progress variable definitions based on $\mathrm{H}_{2}, \mathrm{O}_{2}$ and $\mathrm{H}_{2} \mathrm{O}$ mass fractions (1st-3rd row)

depends on the choice of the initial guess value of the root. In this paper a combined bisection and Newton-Raphson method is used for obtaining $S_{d}^{*}$ according to the NQ extrapolation by solving $\left(S_{d}^{*} / S_{L}\right)^{2} \ln \left(S_{d}^{*} / S_{L}\right)^{2}=-L_{M} K / S_{L}$ once $L_{M}$ is estimated by a non-linear regression method using DNS data. The sensitivity to the initial guess becomes particularly important for large values of $u^{\prime} / S_{L}$. The higher order polynomial in the NE extrapolation is also prone to provide artificial overshoots and undershoots for large values of $u^{\prime} / S_{L}$ and in this respect the N3P extrapolation performs better than the NE extrapolation. It is worth noting that the NQ extrapolation predicts equal magnitudes of positive and negative $S_{d}^{*}$ according to $\left(S_{d}^{*} / S_{L}\right)^{2} \ln \left(S_{d}^{*} / S_{L}\right)^{2}=-L_{M} K / S_{L}$ (see Fig. 3), whereas the distributions of positive and negative $S_{d}^{*}$ are not symmetric according to DNS data. This suggests that it is not straightforward to identify a function $f$ such that $S_{d}^{*}=f(K)$ as different values for $S_{d}^{*}$ can occur for the same value of $K$. An implicit functional relation $f\left(S_{d}^{*}, K\right)$ might circumvent this problem, provided there is a criterion to distinguish the branch of the solution to yield the correct $S_{d}^{*}$.

Based on the performances of the extrapolation relations listed in Table 1, an alternative expression was recently proposed by these authors based on a simple chemistry DNS 
database (Herbert et al. 2020) in the following manner (which will henceforth be referred to as the NEW extrapolation in this paper and Figs. 2-3,5):

$$
S_{d}^{*}=\underbrace{S_{L}\left[1-L_{M}^{\prime} \kappa_{m}+C^{\prime} \kappa_{m}^{2} \delta_{t h}^{2}\right]}_{S_{L s}}-2\left(\rho D_{c} / \rho_{0}\right) \kappa_{m}
$$

where $S_{L}\left[1-L_{M}^{\prime} \kappa_{m}+C^{\prime} \kappa_{m}^{2} \delta_{t h}^{2}\right]$ approximates $\left(S_{r}^{*}+S_{n}^{*}\right)$ and $-2\left(\rho D_{c} / \rho_{0}\right) \kappa_{m}$ is the exact expression for $S_{t}^{*}$. The curvature $\kappa_{m}$ dependence of $\left(S_{r}^{*}+S_{n}^{*}\right)$ remains non-linear for cases A-C for all choices of reaction progress variable, which has been demonstrated in several previous analyses (Chakraborty et al. 2007, 2011a, b; Echekki and Chen 1996; Peters et al. 1998; Chakraborty and Cant 2004, 2005; Chakraborty 2007) and thus is not shown for the sake of conciseness. Thus, a N3P type non-linear extrapolation has been chosen to represent $\left(S_{r}^{*}+S_{n}^{*}\right)$. The variations of the optimum values of $L_{M}^{\prime} / \delta_{t h}$ and $C^{\prime}$ based on a nonlinear regression analysis are shown in Fig. 6 for different values and definitions of $c$ in cases A-C. Figure 6 shows that $L_{M}^{\prime} / \delta_{t h}$ remains small for cases $\mathrm{B}$ and $\mathrm{C}$ for all choices and values of $c$ except for case A where $L_{M}^{\prime} / \delta_{t h}$ assumes a value close to -0.5 for $c=0.8$.
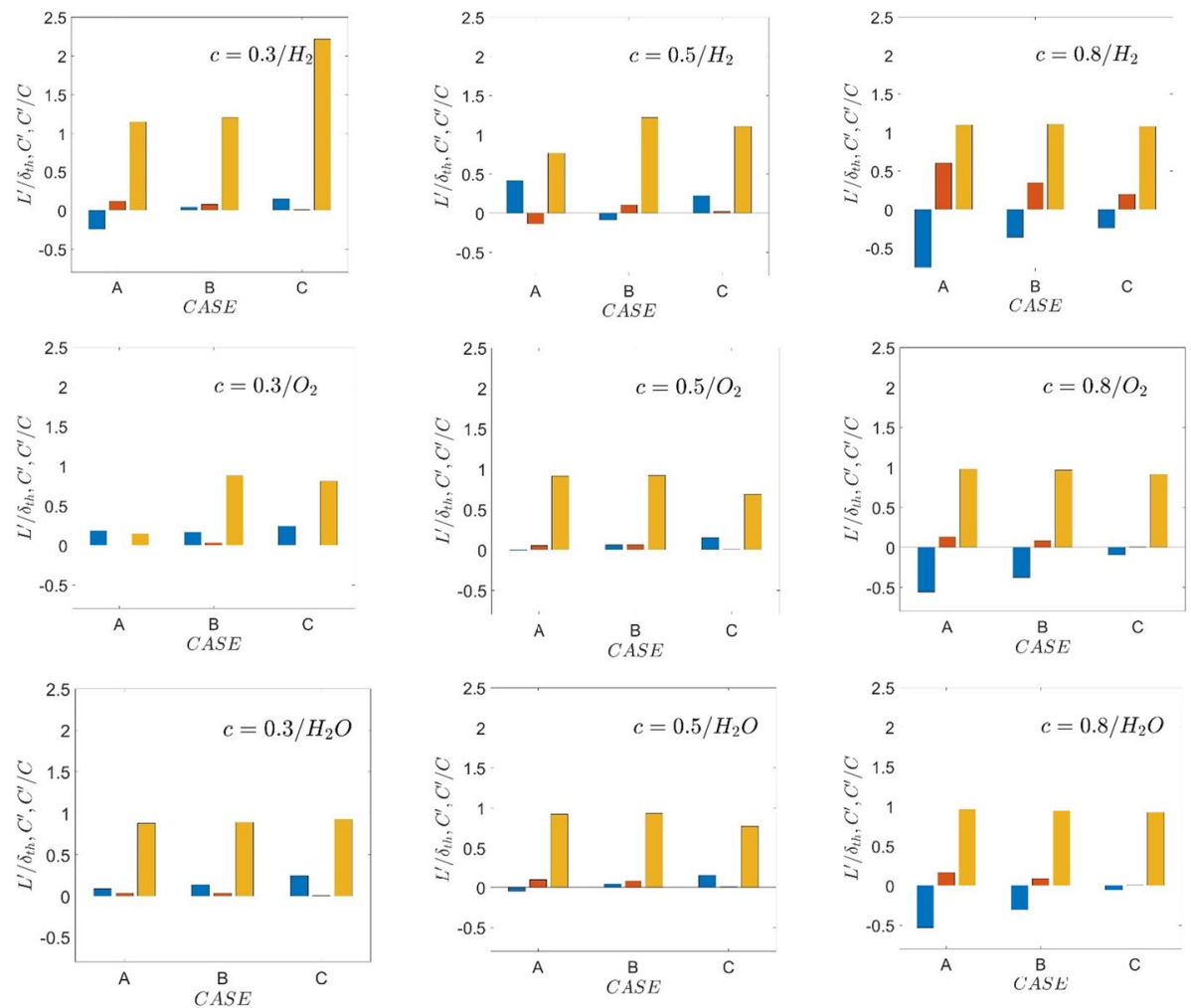

Fig. 6 Variations of the optimised $L_{M}^{\prime} / \delta_{t h}, C^{\prime}$ and $C^{\prime} / C$ for $c=0.3,0.5$ and 0.8 isosurfaces (1st-3rd column) in cases A-C for reaction progress variable definitions based on $\mathrm{H}_{2}, \mathrm{O}_{2}$ and $\mathrm{H}_{2} \mathrm{O}$ mass fractions (1st3rd row) 
However, $L_{M}^{\prime} / \delta_{t h}$ assumes small values for $c=0.3$ and 0.5 for case A. The variation of $C^{\prime}$ has been observed to be qualitatively similar to the corresponding variation of $C$ for the N3P extrapolation (see Fig. 4 ) and the value of $C^{\prime}$ remains close to $C$ (i.e. $C^{\prime} / C \sim 1$ ) for all cases irrespective of the choices and values of $c$ in cases A-C.

The predictions of the NEW extrapolation (i.e. Equation 11) for the $L_{M}^{\prime} / \delta_{\text {th }}$ and $C^{\prime}$ values shown in Fig. 6 are also shown in Figs. 2, 3. The corresponding correlation coefficients are shown in Fig. 5, respectively. Figures 2, 3, 5 indicate that the performance of the NEW relation remains comparable to the N3P model. However, the NEW extrapolation models only the physical contribution of $S_{d}^{*}$ (i.e. $\left(S_{r}^{*}+S_{n}^{*}\right)$ ), which induces the nonlinear curvature dependence of the density-weighted displacement speed. Therefore, it can be expected that the NEW extrapolation might offer less modelling uncertainties in comparison to other alternative extrapolation relations.

\section{Conclusions}

The performances of various extrapolation relations, which approximate the stretch rate and curvature dependences of density-weighted displacement speed, were assessed based on a detailed chemistry DNS database of statistically planar turbulent $\mathrm{H}_{2}$-air premixed flames with an equivalence ratio of 0.7 spanning a range of Karlovitz number values. It was found that density-weighted displacement speed $S_{d}^{*}$ for reaction progress variables defined based on $\mathrm{H}_{2}, \mathrm{O}_{2}$ and $\mathrm{H}_{2} \mathrm{O}$ mass fractions individually, is non-linearly related to both curvature $\kappa_{m}$ and stretch rate $K$, and this trend for stretch rate dependence strengthens with increasing Karlovitz number $K a$. However, the non-linearity of $K$ dependences of $S_{d}^{*}$ is considerably stronger than its curvature $\kappa_{m}$ dependence. It was also found that the extrapolation relation (LS), which expresses $S_{d}^{*}$ as a linear function of stretch rate $K$ does not satisfactorily capture the statistical variation of $S_{d}^{*}$. In contrast, a linear extrapolation relation (LC) in terms of curvature allows for non-linear stretch rate dependence of $S_{d}^{*}$ and thus has been found to be more successful in capturing the statistical behaviours of $S_{d}^{*}$ than the LS extrapolation.

It was found that the non-linear extrapolations (e.g. NQ, NE and N3P) perform better than the linear extrapolation relations (e.g. LC and LS), but the improved performance comes at the cost of additional tuning constants. However, the performance of NQ extrapolation depends on solving a non-linear equation which is sensitive to the choice of initial guess of its root, whereas an extrapolation relation (i.e. NE) which accounts for $\kappa_{m}^{3}$ contribution, is prone to artificial overshoots and undershoots and as a result their performances have been found to be inferior to the N3P extrapolation. Thus, a recently proposed extrapolation relation, which explicitly models curvature dependence of $\left(S_{r}^{*}+S_{n}^{*}\right)$, was found to exhibit promising performance for the range of $\mathrm{Ka}$ considered here. Moreover, the parameters of extrapolation relations (i.e. $L_{M}$ and $C$ ) were found to be sensitive to the choice and definition of $c$ for all extrapolation relations considered here.

Although the non-linear models sometimes show better performance than the LC extrapolation, the performance of the LC extrapolation was found to be remarkably robust, especially from the standpoint of correlation coefficients and does not need any parameter other than the Markstein length. The analysis also demonstrated a non-negligible sensitivity of the Markstein length to the definition of reaction progress variable, which potentially poses a problem for interpretation and comparison of experimental and detailed chemistry 
simulation data. Furthermore, no analytical expressions are available for calculating Markstein length in the context of detailed chemistry data. Comparison of the present data with the earlier simple chemistry analysis reported in Herbert et al. (2020) reveals that the correlation coefficients between density weighted displacement speed and the investigated extrapolation relations are lower for the detailed chemistry case. The foregoing discussion indicates the need for extending classical results of combustion theory in the context of detailed chemistry and transport for the analysis of experimental data, for modelling purposes and for comparison with simulation data where the complexity of chemistry is reduced. The present analysis was conducted for $\mathrm{H}_{2}$-air premixed flames, which behave differently in comparison to hydrocarbon-air premixed flames. Thus, further analysis in this regard will be needed to assess the performances of extrapolation relations for turbulent hydrocarbon-air premixed flames, which will form the basis of future investigations.

Acknowledgements H.G. Im was sponsored by the competitive research funding from King Abdullah University of Science and Technology (KAUST). The DNS were run on the facility at KAUST Supercomputing Laboratory. U. Ahmed and N. Chakraborty gratefully acknowledge EPSRC (grant: EP/P022286/1) for financial support. Further computational support was provided by ARCHER (grant: EP/R029369/1 and EP/ K025163/1), CIRRUS, Leibniz Supercomputing Centre (grant: pn69ga), and HPC facility at Newcastle University (ROCKET).

Funding Open Access funding enabled and organized by Projekt DEAL.

\section{Declaration}

Conflict of interest The authors declare that they have no conflict of interest.

Ethical approval This work did not involve any active collection of human data.

Open Access This article is licensed under a Creative Commons Attribution 4.0 International License, which permits use, sharing, adaptation, distribution and reproduction in any medium or format, as long as you give appropriate credit to the original author(s) and the source, provide a link to the Creative Commons licence, and indicate if changes were made. The images or other third party material in this article are included in the article's Creative Commons licence, unless indicated otherwise in a credit line to the material. If material is not included in the article's Creative Commons licence and your intended use is not permitted by statutory regulation or exceeds the permitted use, you will need to obtain permission directly from the copyright holder. To view a copy of this licence, visit http://creativecommons.org/licenses/by/4.0/.

\section{References}

Ahlgren, P., Jarneving, B., Rousseau, R.: Requirements for a cocitation similarity measure, with special reference to Pearson's correlation coefficient. J. Am Soc. Inf. Sci. Technol. 54, 550-560 (2003)

Boger, M., Veynante, D., Boughanem, H., Trouvé, A.: Direct numerical simulation analysis of flame surface density concept for large eddy simulation of turbulent premixed combustion. Proc. Combust. Inst. 27, 917-925 (1998)

Bray, K.N.C., (1980) Turbulent flows with premixed reactants, In: P.A. Libby, P.A., and F.A.(eds.) Williams, Turbulent Reacting Flows, pp. 115-183, Springer Verlag, Berlin Heidelberg, New York

Brequigny, P., Halter, F., Mounaïm-Roussellea, C.: Lewis number and Markstein length effects on turbulent expanding flames in a spherical vessel. Exp. Therm. Fluid Sci. 73, 33-41 (2016)

Burke, M.P., Chaos, M., Ju, Y., Dryer, F.L., Klippenstein, S.J.: Comprehensive H2-O2 kinetic model for high-pressure combustion. Int. J. Chem. Kin. 44, 444-474 (2012)

Chakraborty, N., Cant, S.: Unsteady effects of strain rate and curvature on turbulent premixed flames in an inflow-outflow configuration. Combust. Flame 137, 129-147 (2004) 
Chakraborty, N., Cant, R.S.: Direct numerical simulation analysis of the flame surface density transport equation in the context of large eddy simulation. Proc. Combust. Inst. 32, 1445-1453 (2009)

Chakraborty, N., Cant, R.S.: Effects of Lewis number on flame surface density transport in turbulent premixed combustion. Combust. Flame 158, 1768-1787 (2011)

Chakraborty, N., Klein, M., Cant, R.S.: Stretch rate effects on displacement speed in turbulent premixed flame kernels in the thin reaction zones regime. Proc. Combust. Inst 31, 1385-1392 (2007)

Chakraborty, N., Hartung, G., Katragadda, M., Kaminski, C.F.: A numerical comparison of 2D and 3D density-weighted displacement speed statistics and implications for laser based measurements of flame displacement speed. Combust. Flame 158, 1372-1390 (2011b)

Chakraborty, N.: Comparison of displacement speed statistics of turbulent premixed flames in the regimes representing combustion in corrugated flamelets and thin reaction zones. Phys. Fluids 19, 105109 (2007)

Chakraborty, N., Cant, R.S.: Influence of Lewis number on curvature effects in turbulent premixed flame propagation in the thin reaction zones regime. Phys. Fluids 17, 105105 (2005)

Chakraborty, N., Klein, M., Cant, R.S.: Effects of turbulent Reynolds number on the displacement speed statistics in the thin reaction zones regime of turbulent premixed combustion. J. Combust. 2011, 473679 (2011a)

Chaudhuri, S., Wu, F., Law, C.K.: Scaling of turbulent flame speed for expanding flames with Markstein diffusion considerations Phys. Rev. E 88, 033005 (2013)

Chen, Z.: On the extraction of laminar flame speed and Markstein length from outwardly propagating spherical flames. Combust. Flame 158, 291-300 (2011)

Chen, J.H., Im, H.G.: Correlation of flame speed with stretch in turbulent premixed methane/air flames. Proc. Combust. Inst 27, 819-826 (1998)

Clavin, P.: Dynamic behavior of premixed flame fronts in laminar and turbulent flows. Prog. Energy Combust. Sci. 11, 1-59 (1985)

Clavin, P., Joulin, G.: Premixed flames in large scale and high intensity turbulent flow. J. Phys. Lett. 44, L1-L12 (1983)

Dave, H., Chaudhuri, S., S. : Evolution of local flame displacement speeds in turbulence. J. Fluid Mech. 884, A46 (2020). https://doi.org/10.1017/jfm.2019.896

Dopazo, C., Cifuentes, L., Martin, J., Jimenez, C.: Strain rates normal to approaching iso-scalar surfaces in a turbulent premixed flame. Combust. Flame 162, 1729-1736 (2015)

Echekki, T., Chen, J.H.: Unsteady strain rate and curvature effects in turbulent premixed methane-air flames. Combust. Flame 106, 184-202 (1996)

Frankel, M.L., Sivashinsky, G.I.: On effects due to thermal expansion and lewis number in spherical flame propagation. Combust. Sci. Technol. 31, 131-138 (1983)

Giannakopoulos, G.K., Gatzoulis, A., Frouzakis, E., Matalon, M., Tomboulides, A.G.: Consistent definitions of "Flame Displacement Speed" and "Markstein Length" for premixed flame propagation. Combust. Flame 162, 1249-1264 (2015)

Hawkes, E.R., Cant, R.S.: Implications of a flame surface density approach to large eddy simulation of premixed turbulent combustion. Combust. Flame 126, 1617-1629 (2001)

Herbert, A., Ahmed, U., Chakraborty, N., Klein, M.: Applicability of extrapolation relations for curvature and stretch rate dependences of displacement speed for statistically planar turbulent premixed flames. Combust. Theor. Modell. (2020). https://doi.org/10.1080/13647830.2020.1802066

Hun, I., Huh, K.Y.: Roles of displacement speed on evolution of flame surface density for different turbulent intensities and Lewis numbers in turbulent premixed combustion. Combust. Flame 152, 194-205 (2008)

Im, H.G., Chen, J.H.: Preferential diffusion effects on the burning rate of interacting turbulent premixed hydrogen-air flames. Combust. Flame 126, 246-258 (2002)

Im, H.G., Arias, P.G., Chaudhuri, S., Uranakara, H.A.: Direct numerical simulations of statistically stationary turbulent premixed flames. Combust. Sci. Technol. 188, 1182-1198 (2016)

Istratov, A.G., Librovich, V.B.: On the stability of gasdynamic discontinuities associated with chemical reaction. the case of a spherical flame. Astronautica Acta 14, 453-467 (1969)

Karlovitz, B., Denniston, D.W., Knapschaefer, D.H., Wells, F.E.: Studies of turbulent flames. 4th Symposium (International) on combustion. Baltimore: Williams and Wilkins 4(1), 613-620 (1953). https:// doi.org/10.1016/S0082-0784(53)80082-2

Karpov, V.P., Lipatnikov, A.N., Wolanski, P.: Finding the Markstein number using the measurements of expanding spherical laminar flames. Combust. Flame 109, 436-448 (1997)

Kelley, A.P., Law, C.K.: Nonlinear effects in the extraction of laminar flame speeds from expanding spherical flames. Combust. Flame 156, 1844-1851 (2009) 
Kelley, A.P., Bechtold, J.K., Law, C.K.: Premixed flame propagation in a confining vessel with weak pressure rise. J. Fluid Mech. 691, 26-51 (2011)

Klein, M., Kasten, C., Chakraborty, N., Mukhadiyev, N., Im, H.G.: Turbulent scalar fluxes in Hydrogen-Air premixed flames at low and high Karlovitz numbers. Combust. Theor. Modell. 22, 1033-1048 (2018)

Klimov, A.M.: Laminar flame in a turbulent flow. Zhournal Prikladnoi Mekchaniki i Tekhnicheskoi Fiziki 3, 49-58 (1963)

Lipatnikov, A.N., Chomiak, J.: Molecular transport effects on turbulent flame propagation and structure. Prog. Energy Combust. Sci. 31, 1-73 (2005)

Lipatnikov, A.N., Shy, S.S., Li, W.: Experimental assessment of various methods of determination of laminar flame speed in experiments with expanding spherical flames with positive Markstein lengths. Combust. Flame 162, 2840-2854 (2015)

Markstein, G.H.: Experimental and theoretical studies of flame front stability. J Aeronaut Sci 18, 199-209 (1951)

Matalon, M., Matkowsky, B.J.: Flames as gas dynamic discontinuities. J Fluid Mech 124, 239-260 (1982)

Papapostolou, V., Wacks, D.H., Klein, M., Chakraborty, N., Im, H.G.: Enstrophy transport conditional on local flow topologies in different regimes of premixed turbulent combustion. Sci. Rep. 7, 11545 (2017)

Passot, T., Pouquet, P.: Numerical simulation of compressible homogeneous flows in the turbulent regime. J. Fluid Mech. 181, 441-466 (1987)

Pera, C., Chevillard, S., Reveillon, R.: Effects of residual burnt gas heterogeneity on early flame propagation and on cyclic variability in spark-ignited engines. Combust. Flame 160, 1020-1032 (2013)

Peters, N.: Turbulent Combustion, 1st edn. Cambridge University Press (2000)

Peters, N., Terhoeven, P., Chen, J.H., Echekki, T.: Statistics of flame displacement speeds from computations of 2-D unsteady methane-air flames. Proc. Combust. Inst. 27, 833-839 (1998)

Pope, S.B.: The evolution of surfaces in turbulence. Int. J. Engg. Sci. 26, 445-469 (1988)

Reddy, H., Abraham, J.: Two-dimensional direct numerical simulation evaluation of the flame-surface density model for flames developing from an ignition kernel in lean methane/air mixtures under engine conditions. Phys. Fluids 24, 105108 (2012)

Rogallo, R.S.: Numerical experiments in homogeneous turbulence, NASA Ames Research Center Report No. 81315. (1981)

Ronney, P.D., Sivashinsky, G.I.: A theoretical study of propagation and extinction of nonsteady spherical flame fronts. SIAM J. Appl. Math. 49, 1029-1046 (1989)

Sabelnikov, V., Lipatnikov, A.N., Chakraborty, N., Nishiki, S., Hasagawa, T.: A balance equation for the mean rate of product creation in premixed turbulent flames. Proc. Combust. Inst. 36, 1893-1901 (2017)

Venkateswaran, P., Marshall, A., Seitzman, J., Lieuwen, T.: Scaling turbulent flame speeds of negative Markstein length fuel blends using leading points concepts. Combust. Flame. 162(2), 375-387 (2015). https://doi.org/10.1016/j.combustflame.2014.07.028

Wacks, D.H., Chakraborty, N., Klein, M., Arias, P.G., Im, H.G.: Flow topologies in different regimes of premixed turbulent combustion: A direct numerical simulation analysis. Phys. Rev. F 1, 083401 (2016)

Wu, C.K., Law, C.K.: On the determination of laminar flame speeds from stretched flames. Proc. Combust. Inst. 20, 1941-1949 (1984)

Wu, F., Liang, W., Chen, Z., Ju, Y., Law, C.K.: Uncertainty in stretch extrapolation of laminar flame speed from expanding spherical flames. Proc. Combust. Inst. 35, 663-670 (2005)

Yoo, C.S., Wang, Y., Trouvé, A., Im, H.G.: Characteristic boundary conditions for direct simulations of turbulent counterflow flames. Combust. Theor. Model. 9, 617-646 (2005)

Publisher's Note Springer Nature remains neutral with regard to jurisdictional claims in published maps and institutional affiliations. 


\section{Authors and Affiliations}

Nilanjan Chakraborty ${ }^{1} \cdot$ Alexander Herbert $^{2} \cdot$ Umair Ahmed ${ }^{1} \cdot$ Hong G. Im $^{3}$. Markus Klein ${ }^{2}$ (D)

Nilanjan Chakraborty

nilanjan.chakraborty@ncl.ac.uk

Hong G. Im

hong.im@kaust.edu.sa

1 School of Engineering, Newcastle University, Claremont Road, Newcastle-Upon-Tyne NE1 7RU, UK

2 Department of Aerospace Engineering, Bundeswehr University Munich, Werner-Heisenberg-Weg 39, 85577 Neubiberg, Germany

3 Clean Combustion Research Center, King Abdullah University of Science and Technology (KAUST), Thuwal 23955-6900, Saudi Arabia 